\title{
Diet in Wound Care: Can Nutrition Impact Healing?
}

\author{
Bridget E. Shields, MD
}

\section{PRACTICE POINTS}

- Optimizing wound healing requires local and systemic therapies as well as adequate nutritional support.

- Malnutrition is a potentially modifiable risk factor that may contribute to impaired wound healing.

- Patients with chronic wounds and specific nutrient deficiencies should supplement to optimize healing.

- Evidence-based recommendations surrounding the role of dietary supplementation and nutritional support in patients without deficiencies for wound healing are lacking and should be tailored to individual patients.
Wound healing requires a multifaceted approach that often includes topical and oral therapies, adjustment of mechanical factors, and behavioral and lifestyle modifications. Although the causes of wounds encountered in dermatology vary extensively, the importance of nutrition underlies all wound healing. This article reviews the existing literature on macronutrient and micronutrient supplementation to clarify the potentially complementary role for nutritional support in chronic wounds.

Cutis. 2021;108:325-328.

$\square$ ermatologists commonly manage a variety of wounds in the outpatient setting. Wound healing requires a multifaceted approach that often includes topical and oral therapies, adjustment of mechanical factors, and behavioral and lifestyle modifications. Physiologically, wound healing requires an inflammatory phase, a proliferative phase, and a remodeling phase. Chronic wounds undergo a prolonged inflammatory response hindered by decreased growth factors and increased wound bioburden. Malnutrition has been routinely associated with wound chronicity and serves as a modifiable risk factor that may improve wound healing outcomes. ${ }^{2}$

Although the causes of wounds encountered in dermatology vary extensively, the importance of nutrition underlies all wound healing. Caloric needs in wound healing have been estimated at 30 to $40 \mathrm{kcal} / \mathrm{kg}$ dependent on baseline body weight, age, medical comorbidities, activity level, stage of wound healing, wound size, and number of wounds. ${ }^{1,3,4}$ Nutritional supplementation is patient dependent, but this article serves to review the existing literature on macronutrient and micronutrient supplementation to clarify the potentially complementary role for nutritional support in chronic wounds. All patients should be screened with a thorough history, review of systems, and physical examination for existing nutrient deficiencies. Patients with age-related or chronic diseases are at increased risk for nutritional deficiency, and focused laboratory testing may be warranted. Supplementation for specific deficiencies with help from a registered dietician is recommended.

\section{Macronutrients for Wound Healing}

Protein-Protein is the most widely known macronutrient required for wound healing. The primary function of dietary protein is to provide amino acids to perform physiologic functions. ${ }^{5}$ Not only does cutaneous injury increase the metabolic needs of the wounded area, but large amounts of protein can be continually lost through wound exudates. Protein is necessary for the immune response required to transition from inflammatory to proliferative phases of wound healing. ${ }^{6}$ Protein energy deficiency has been reported to reduce fibroblast activity,

From the Department of Dermatology, University of Wisconsin School of Medicine and Public Health, Madison.

The author reports no conflict of interest.

Correspondence: Bridget E. Shields, MD, 1 S Park St, University of Wisconsin School of Medicine and Public Health, Department of Dermatology,

Madison, WI 53711 (bshields@dermatology.wisc.edu).

doi: 10.12788 /cutis.0407 
delay angiogenesis, and decrease collagen formation. ${ }^{7}$ Additionally, protein is required for the formation of inflammatory cells and maintenance of oncotic pressure, specifically in venous insufficiency wounds. ${ }^{1}$

The current recommended dietary allowance for protein in healthy adults is $0.8 \mathrm{~g} / \mathrm{kg}$ daily of body weight. In patients with pressure ulcerations, a goal recommended dietary allowance of 1.25 to $2.0 \mathrm{~g} / \mathrm{kg}$ daily of body weight, dependent on ulceration size, has been recommended by the National Pressure Ulcer Advisory Panel and European Pressure Ulcer Advisory Panel. ${ }^{8}$ This recommendation was based on a series of studies that reported enhanced healing rates in patients with pressure ulcers receiving higher-protein diets. ${ }^{9}$ The largest study to date was double-blinded and included 89 residents of long-term care facilities with stage II to stage IV pressure ulcers. ${ }^{10}$ Participants were randomized to receive commercial protein supplementation vs placebo. At the end of 8 weeks, a statistically significant difference was seen in mean (SD) pressure ulcer scale for healing scores (3.55 [4.66] vs 3.22 [4.11]; P<.05)..$^{10}$ A 2014 Cochrane review failed to identify benefit associated with nutritional interventions for either the prevention and/or treatment of pressure ulcers. ${ }^{11}$ Specific recommendations on protein intake for other types of chronic wounds have not been proposed. Protein supplementation generally is provided orally, if tolerated. Liquid supplements such as Boost (Nestlé), Carnation Breakfast Essentials (Nestlé), NuBasics (SupremeMed), Resource (Nestlé Health Science), and Ensure (Abbott Laboratories) are frequently used to supplement both protein and caloric intake. Protein oversupplementation has not been associated with improved outcomes and may cause or exacerbate other medical comorbidities.

\section{Fatty Acids for Wound Healing}

Wound healing is an anabolic process that requires adequate intake of substrates such as glucose and fat. Carbohydrates serve as the major energy source required for wound healing, while fats are thought to play roles in cell membrane development and modulation of cellular signaling. ${ }^{1}$ Fats utilize a unique pathway for energy production through beta-oxidation and the production of adenosine triphosphate, allowing available protein to be harnessed for wound healing. ${ }^{1}$ Omega-3 and omega-6 fatty acids serve as precursors to prostaglandins, leukotrienes, and thromboxane-all key mediators of the inflammatory phase of wound healing. ${ }^{3}$ Omega- 3 fatty acids are thought to downregulate genes involved in proinflammatory pathways, ${ }^{12}$ as well as to diminish lymphocyte proliferation and levels of IL-1 $\beta$, tumor necrosis factor $\alpha$, and IL-6 in vitro. ${ }^{13}$ In vivo studies assessing the impact of omega-3 fatty acid supplementation on wound healing are minimal, and the role of dietary supplementation for this indication remains unknown. Fish oil contains the omega-3 fatty acid-rich eicosapentaenoic acid and docosahexaenoic acid, which has been compared to mineral oil supplementation for wound healing in healthy adults. When fish oil was supplemented for 4 weeks, no significant differences were identified in time to complete wound healing between groups. Interestingly, significantly higher levels of the proinflammatory cytokine IL-1 $\beta$ were identified in blister fluid at 24 hours after blistering vs the placebo group $(\mathrm{t}=2.52, \mathrm{df}=25, P<.05) .{ }^{14}$ Prior studies evaluating wound healing in animal models similarly identified longer times to re-epithelialization after omega-3 polyunsaturated fatty acid supplementation orally and topically. ${ }^{15,16}$ The fatty acid quality and composition consumed also may impact wound healing, as high-fat diets that are not rich in omega- 3 fatty acids have been shown to promote inflammation and impair wound healing in rats, but this has not been thoroughly explored in human trials. ${ }^{17}$ Although adequate intake of these macronutrients is important, excessive intake may be harmful. Larger prospective trials are needed to shed light on the dose and composition of fatty acid supplementation that may optimize wound healing.

\section{Vitamins and Micronutrients Required for Wound Healing}

Vitamin A-Many vitamins serve as cofactors for the enzymatic processes required in wound healing. Vitamin A is an essential fat-soluble vitamin that serves a variety of dermatologic functions and promotes wound healing through stimulation of fibroblasts and ground substance, and it facilitates epithelial cell differentiation when applied topically., ${ }^{3,18}$ Vitamin A works through the activation of retinoid receptors on endothelial cells, fibroblasts, keratinocytes, melanocytes, and sebocytes, and has purported anti-inflammatory effects that aid the healing of open wounds. ${ }^{3}$ Additionally, vitamin A is thought to enhance cytokine release in the inflammatory phase of wound healing. ${ }^{19}$ Supplemental vitamin A has been associated with positive effects on acute wound healing, burns, and radiation injuries. ${ }^{3}$ The utility of vitamin A supplementation in chronic wounds remains unknown; however, it has been shown to be beneficial in patients with inflammatory disease, such as rheumatoid arthritis, on corticosteroid therapy. Vitamin A supplementation in this population has been shown to counteract the negative effects of corticosteroids on wound healing via downregulation of transforming growth factor $\beta$ and insulinlike growth factor $1 .{ }^{20}$ Vitamin A deficiency has been associated with impaired progression through inflammatory and remodeling phases of healing due to altered B-cell and T-cell function and antibody production. ${ }^{1}$ Some experts recommend short courses of oral vitamin A supplementation to enhance wound healing at doses between 10,000 and 25,000 IU daily. ${ }^{2,3}$ Large, populationbased studies are needed, and the safety supporting this recommendation in all patients remains unknown.

Vitamin C-Vitamin C is widely known for its role in collagen formation, immunomodulation, and antioxidant capacity. ${ }^{1}$ Although vitamin C deficiency is associated with decreased collagen synthesis and impaired wound 
healing, ${ }^{21}$ the utility of long-term supplementation in patients who are not deficient remains unexplored. A systematic review evaluating interventional studies utilizing vitamin $C$ supplementation on pressure ulcerations and surgical wound healing concluded that convincing evidence exists only for supplementation with at least $500 \mathrm{mg}$ of vitamin C. The authors noted, "There is little evidence for improved healing of surgical wounds by high-dose single vitamin $C$ supplementation (1-3 g/day)." ${ }^{22}$ In a prospective, randomized, controlled trial, 20 patients with pressure ulcerations were supplemented with vitamin C vs placebo with a mean reduction in pressure-sore area of $84 \%$ after 1 month in the vitamin C-supplemented group compared to $42.7 \%$ in the placebo group $(P<.005)$. A limitation of this study is the small population. ${ }^{23}$ One current recommendation for vitamin $C$ supplementation in chronic wounds is for $500 \mathrm{mg}$ daily in uncomplicated wounds to $2 \mathrm{~g}$ daily in severe wounds. ${ }^{3}$ Additional studies have suggested that the benefits of vitamin $\mathrm{C}$ supplementation are maximized when given in combination with zinc and arginine..$^{22}$ At this time, evaluation for vitamin C deficiency and appropriate supplementation in patients with chronic wounds is needed.

Zinc-Minerals similarly play important roles in enzymatic regulation. Hundreds of zinc-containing enzymes are involved in wound healing and are required in tissue repair, growth, antioxidant capacity, and immune function. ${ }^{1,24}$ Zinc is specifically critical to collagen, DNA, RNA, and protein synthesis, as well as cellular proliferation. ${ }^{4}$ Zinc deficiency has been encountered in the setting of chronic wounds with extensive drainage, decreased dietary intake, or excessive gastrointestinal losses. ${ }^{25}$ Although many studies exist evaluating the utility of zinc supplementation on wound healing, many are confounded by multinutrient supplementation. No studies to date support zinc supplementation when zinc deficiency is absent. Patient assessment for medications or conditions that may impact zinc metabolism should be completed. Importantly, zinc supplementation can interfere with the absorption of other cations, so excessive supplementation should be avoided. ${ }^{1}$

\section{Amino Acids for Wound Healing}

Arginine-Arginine is an essential amino acid that serves as a substrate for cellular proliferation, collagen deposition, and lymphocyte function. ${ }^{8,26,27}$ Arginine serves as the biologic precursor for nitric oxide (NO), a substrate that has important wound healing properties. Nitric oxide metabolites have been shown to positively regulate wound repair while NO metabolites are reduced in wound environments in diabetic ulcerations. ${ }^{28,29}$ Arginine also is a proline precursor, an essential building block for collagen synthesis, ${ }^{6,30}$ and a stimulator of growth hormone and T cells. . $^{30,31}$ Animal studies have suggested L-arginine supplementation may reverse impaired NO synthesis in diabetic wounds. ${ }^{28} \mathrm{~A}$ single randomized trial assessing differing doses of arginine supplementation on stage II or stage IV pressure ulcers noted an almost twofold improvement in healing time. ${ }^{32}$ However, human studies have not shown increased rates of re-epithelialization of skin graft donor sites when provided oral or parenteral arginine supplementation. ${ }^{33}$ Inadequate data currently exist to support regular arginine supplementation for all types of wounds, and no safe dose of daily arginine intake has been established.

Glutamine-Similarly, glutamine supplementation has been proposed to accelerate wound healing due to its role as a primary metabolic fuel source for rapidly proliferating cells such as epithelial cells and fibroblasts. ${ }^{8}$ Glutamine is thought to induce expression of heat-shock proteins and protect against inflammatory and infectious wound complications ${ }^{34}$ Additionally, glutamine is thought to increase tissue insulin sensitivity, which may prove beneficial in wounds, as topical insulin previously has been shown in animal and human models to promote healing. ${ }^{35}$ Glutamine is thought to play a role in the inflammatory phase of wound healing via superoxide production, leukocyte apoptosis, and phagocytosis. ${ }^{6,34,36}$ Unfortunately, numerous randomized trials on glutamine supplementation have resulted in conflicting evidence confounded by multisupplementation within the same trial. ${ }^{37,38}$ A doubleblind, randomized, controlled trial of 270 participants assessed the effect of oral supplementation with arginine, glutamine, or $\beta$-hydroxy- $\beta$-methylbutyrate vs control in the healing time of diabetic foot ulcerations. Significant differences in wound closure time at week 16 were only identified in participants with low albumin levels $(\leq 40 \mathrm{~g} / \mathrm{L})$ who were supplemented $(50.8 \%)$ vs the control group (34.9\%; $P=.0325)$ and in those with poor limb perfusion (ankle-brachial index of $<1.0$ ) who were supplemented $(60.3 \%)$ vs the control group (39.3\%; $P=.0079) .{ }^{39}$ Ongoing clinical trials evaluating the effects of glutamine supplementation on differing wound types will hopefully shed light on the efficacy of supplementation.

\section{Final Thoughts}

Wound healing is multifactorial and should consider the health status and medical comorbidities of each patient treated. We propose an individualized approach to wound healing that includes exploration of specific macronutrient and micronutrient deficiencies, as malnutrition has been associated with wound chronicity and serves as a modifiable risk factor to improve healing. ${ }^{2}$ The evidence backing specific nutrient supplementation in patients with deficiencies is strong and should be considered in patients with chronic wounds. Adequate caloric intake and protein content should be recommended for most wound patients; however, excessive protein intake has not been beneficial in wound healing. The data behind specific amino acid and vitamin supplementation are limited at this time. As with other therapeutics, there is likely an appropriate dose for supplementation that has not yet been elucidated. Consideration of wound type, size, depth, exudate, and underlying cause are important to 
optimize healing and tailor nutritional supplementation to each patient. We hope future studies will illuminate the complementary role of dietary intake and nutrient supplementation for the treatment of chronic nonhealing wounds.

\section{REFERENCES}

1. Quain AM, Khardori NM. Nutrition in wound care management: a comprehensive overview. Wounds. 2015;27:327-335

2. Stechmiller JK. Understanding the role of nutrition and wound healing. Nutr Clin Pract. 2010;25:61-68. doi:10.1177/0884533609358997

3. Molnar JA, Underdown MJ, Clark WA. Nutrition and chronic wounds. Adv Wound Care (New Rochelle). 2014;3:663-681. doi:10.1089 /wound.2014.0530

4. Dorner B, Posthauer ME, Thomas D; Panel NPUA. The role of nutrition in pressure ulcer prevention and treatment: National Pressure Ulcer Advisory Panel white paper. Adv Skin Wound Care. 2009;22:212-221. doi:10.1097/01.ASW.0000350838.11854.0a

5. Collins N. Protein and wound healing. Adv Skin Wound Care. 2001;14:288-289. doi:10.1097/00129334-200111000-00008

6. Barchitta M, Maugeri A, Favara G, et al. Nutrition and wound healing: an overview focusing on the beneficial effects of curcumin [published online March 5, 2019]. Int J Mol Sci. doi:10.3390/ijms20051119

7. Harris CL, Fraser C. Malnutrition in the institutionalized elderly: the effects on wound healing. Ostomy Wound Manage. 2004;50:54-63.

8. Saghaleini SH, Dehghan K, Shadvar K, et al. Pressure ulcer and nutrition. Indian J Crit Care Med. 2018;22:283-289. doi:10.4103/ijccm .IJCCM_277_17

9. Breslow RA, Hallfrisch J, Guy DG, et al. The importance of dietary protein in healing pressure ulcers. J Am Geriatr Soc. 1993;41:357-362. doi:10.1111/j.1532-5415.1993.tb06940.x

10. Lee SK, Posthauer ME, Dorner B, et al. Pressure ulcer healing with a concentrated, fortified, collagen protein hydrolysate supplement: a randomized controlled trial. Adv Skin Wound Care. 2006;19:92-96 doi:10.1097/00129334-200603000-00011

11. Langer G, Fink A. Nutritional interventions for preventing and treating pressure ulcers. Cochrane Database Syst Rev. 2014;6:CD003216. doi:10.1002/14651858.CD003216.pub2

12. Bouwens $M$, van de Rest $\mathrm{O}$, Dellschaft $\mathrm{N}$, et al. Fish-oil supplementation induces antiinflammatory gene expression profiles in human blood mononuclear cells. Am J Clin Nutr. 2009;90:415-424. doi:10.3945 lajen.2009.27680

13. Meydani SN, Endres S, Woods MM, et al. Oral (n-3) fatty acid supplementation suppresses cytokine production and lymphocyte proliferation: comparison between young and older women. J Nutr. 1991;121:547-555. doi:10.1093/jn/121.4.547

14. McDaniel JC, Belury M, Ahijevych K, et al. Omega-3 fatty acids effect on wound healing. Wound Repair Regen. 2008;16:337-345. doi:10.1111 /j.1524-475X.2008.00388.x

15. Mooney MA, Vaughn DM, Reinhart GA, et al. Evaluation of the effects of omega-3 fatty acid-containing diets on the inflammatory stage of wound healing in dogs. Am J Vet Res. 1998;59:859-863.

16. Cardoso CR, Souza MA, Ferro EA, et al. Influence of topical administration of n-3 and n-6 essential and n-9 nonessential fatty acids on the healing of cutaneous wounds. Wound Repair Regen. 2004;12:235-243. doi:10.1111/j.1067-1927.2004.012216.x

17. Rosa DF, Sarandy MM, Novaes RD, et al. High-fat diet and alcohol intake promotes inflammation and impairs skin wound healing in Wistar rats. Mediators Inflamm. 2018;2018:4658583. doi:10.1155/2018/4658583

18. Levenson SM, Gruber CA, Rettura G, et al. Supplemental vitamin A prevents the acute radiation-induced defect in wound healing. Ann Surg. 1984;200:494-512. doi:10.1097/00000658-198410000-00011

19. Palmieri B, Vadalà M, Laurino C. Nutrition in wound healing: investigation of the molecular mechanisms, a narrative review. J Wound Care. 2019;28:683-693. doi:10.12968/jowc.2019.28.10.683
20. Ehrlich HP, Hunt TK. Effects of cortisone and vitamin A on wound healing. Ann Surg. 1968;167:324-328. doi:10.1097/00000658 -196803000-00004

21. Pullar JM, Carr AC, Vissers MCM. The roles of vitamin C in skin health [published online August 12, 2017]. Nutrients. doi:10.3390/nu9080866

22. Ellinger S, Stehle P. Efficacy of vitamin supplementation in situations with wound healing disorders: results from clinical intervention studies. Curr Opin Clin Nutr Metab Care. 2009;12:588-595. doi:10.1097 /MCO.0b013e328331a5b5

23. Taylor TV, Rimmer S, Day B, et al. Ascorbic acid supplementation in the treatment of pressure-sores. Lancet. 1974;2:544-546. doi:10.1016 /s0140-6736(74)91874-1

24. Ibs KH, Rink L. Zinc-altered immune function. J Nutr. 2003;133 (5 suppl 1):1452S-1456S. doi:10.1093/jn/133.5.1452S

25. Hoffman M, Micheletti RG, Shields BE. Nutritional dermatoses in the hospitalized patient. Cutis. 2020;105:296-302, 308, E1-E5.

26. Chow O, Barbul A. Immunonutrition: role in wound healing and tissue regeneration. Adv Wound Care (New Rochelle). 2014;3:46-53. doi:10.1089/wound.2012.0415

27. Singh $\mathrm{K}$, Coburn LA, Barry DP, et al. L-arginine uptake by cationic amino acid transporter 2 is essential for colonic epithelial cell restitution. Am J Physiol Gastrointest Liver Physiol. 2012;302:G1061-G1073. doi:10.1152/ajpgi.00544.2011

28. Witte MB, Thornton FJ, Tantry U, et al. L-Arginine supplementation enhances diabetic wound healing: involvement of the nitric oxide synthase and arginase pathways. Metabolism. 2002;51:1269-1273. doi:10.1053/meta.2002.35185

29. Witte MB, Barbul A. Role of nitric oxide in wound repair. Am J Surg. 2002;183:406-412. doi:10.1016/s0002-9610(02)00815-2

30. Barbul A. Proline precursors to sustain Mammalian collagen synthesis. J Nutr. 2008;138:2021S-2024S. doi:10.1093/jn/138.10.2021S

31. Wu G, Bazer FW, Davis TA, et al. Arginine metabolism and nutrition in growth, health and disease. Amino Acids. 2009;37:153-168. doi:10.1007 /s00726-008-0210-y

32. Leigh B, Desneves $\mathrm{K}$, Rafferty J, et al. The effect of different doses of an arginine-containing supplement on the healing of pressure ulcers. J Wound Care. 2012;21:150-156. doi:10.12968 /jowc.2012.21.3.150

33. Debats IB, Koeneman MM, Booi DI, et al. Intravenous arginine and human skin graft donor site healing: a randomized controlled trial. Burns. 2011;37:420-426. doi:10.1016/j.burns. 2010.06.003

34. Wischmeyer PE. Glutamine and heat shock protein expression. Nutrition. 2002;18:225-228. doi:10.1016/s0899-9007(01)00796-1

35. Wang J, Xu J. Effects of topical insulin on wound healing: a review of animal and human evidences. Diabetes Metab Syndr Obes. 2020;13:719727. doi:10.2147/DMSO.S237294

36. Newsholme P. Why is L-glutamine metabolism important to cells of the immune system in health, postinjury, surgery or infection? J Nutr. 2001;131(9 suppl):2515S-2522S; discussion 2523S-2524S. doi:10.1093/jn/131.9.2515S

37. Aquino VM, Harvey AR, Garvin JH, et al. A double-blind randomized placebo-controlled study of oral glutamine in the prevention of mucositis in children undergoing hematopoietic stem cell transplantation: a pediatric blood and marrow transplant consortium study. Bone Marrow Transplant. 2005;36:611-616. doi:10.1038/sj.bmt.1705084

38. Ward E, Smith M, Henderson M, et al. The effect of high-dose enteral glutamine on the incidence and severity of mucositis in paediatric oncology patients. Eur J Clin Nutr. 2009;63:134-140. doi:10.1038/sj.ejcn.1602894

39. Armstrong DG, Hanft JR, Driver VR, et al. Effect of oral nutritional supplementation on wound healing in diabetic foot ulcers: a prospective randomized controlled trial. Diabet Med. 2014;31:1069-1077. doi:10.1111/dme.12509 